\title{
A prokaryotic phytochrome
}

Dhytochrome photoreceptors are almost certainly ubiquitous in green plants, regulating numerous aspects of development throughout their life cycle. Phytochromes were thought to exist only in plants, but the recently described sequence of the chromosome from the cyanobacterium Synechocystis revealed a gene that seemed to encode a phytochrome-like protein ${ }^{1}$. By expressing this gene in Escherichia coli and feeding appropriate chromophores we show that it encodes a phytochrome, which may offer an excellent starting material for crystallization and X-ray diffraction analysis.

The carboxy-terminal amphiphilic structure of phytochrome resembles that of bacterial sensory histidine-kinases, a group of enzymes used by prokaryotes to monitor and react to various aspects of their environment $^{2-4}$. Conceptual translation of the Synechocystis sp. PCC 6803 open reading frame slr0473 (the putative Synechocystis phy gene) yields a product that shows similarity to plant phytochromes throughout its length and to bacterial sensory kinases towards the $\mathrm{C}$ terminus ${ }^{1,5}$. In particular, the chromophore-binding domain, highly conserved in all phytochromes, is clearly represented in the product (residues Val 246-Asp 280). This, however, does not prove that the gene product is a genuine phytochrome. Phycocyanin levels prevent spectral photoreversibility measurements ${ }^{6,7}$ of phytochrome in cyanobacteria, so we investigated the puta-

$$
\text { a }
$$

$M_{\mathrm{r}(\mathrm{K})} 1 \quad 2 \quad 3$

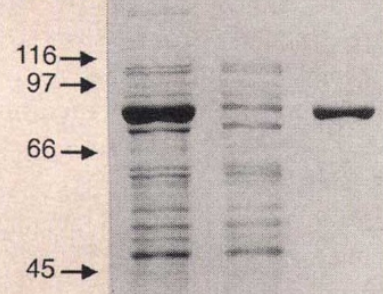

$29 \rightarrow$

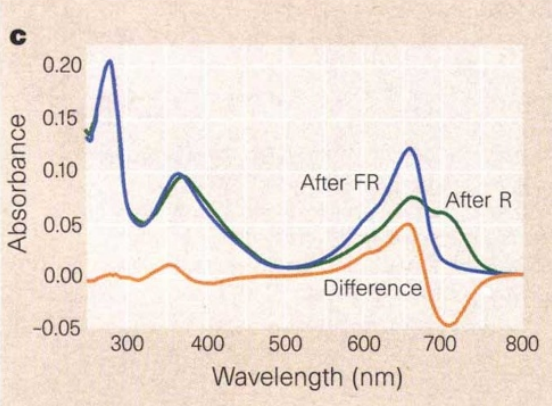

tive phy gene product by expression-cloning in E. coli using the vector PQE12.

Expression of the Synechocystis PHY apoprotein was very efficient. Products of relative molecular mass 85,000 accumulated to about $50 \%$ total soluble protein (Fig. 1a, lane 1). This contrasts with results from similar approaches with plant phytochrome genes in $E$. coli which are usually very weakly expressed or give rise to largely insoluble products $^{8-10}$. The clone was engineered to express a C-terminal polyhistidine tag for nickel-affinity purification. The product bound quantitatively to Ni-NTA (lane 2) and was eluted as a homogeneous apoprotein (lane 3), which could be concentrated to a 5-10 $\mathrm{mg} \mathrm{ml}^{-1}$ solution.

Plant phytochrome apoproteins auto catalytically attach linear tetrapyrrole chromophores such as phycocyanobilin (PCB) ${ }^{11}$, abundant in the cytoplasm of cyanobacteria. Indeed, the Synechocystis PHY apoprotein attached purified $\mathrm{PCB}$, producing visibly photochromic holoprotein ( $\mathrm{phy}^{*}$, Fig. 1b). In contrast, plant phytochromes expressed in E. coli show poor autoassembly, folding incorrectly ${ }^{8,9,12}$. Synechocystis phy ${ }^{*}$ was analysed spectrophotometrically after exposure to saturating monochromatic 657 $\mathrm{nm}$ (red) and $731 \mathrm{~nm}$ (far-red) irradiation (Fig. 1c). The spectra are reminiscent of plant phytochrome-PCB adducts ${ }^{11}$ with absorb-ance maxima at 658 and $702 \mathrm{~nm}$ after red and far-red irradiation, respec-

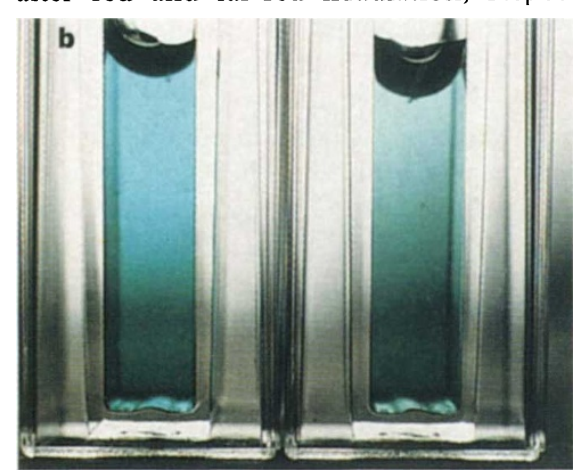

Figure $1 \mathrm{a}$, Expression and affinity purification of recombinant PHY apoprotein in E. coli. SDSPAGE $10 \%$ gel stained with Coomassie. Lane 1, total soluble protein in lysate; 2 , after adsorption to Ni-NTA matrix; 3, $250 \mathrm{mM}$ imidazole eluate. b, Photochromicity of phy ${ }^{\star}$ holoprotein. Stoichiometric amounts of $\mathrm{PCB}$ were added to PHY (3 $\left.\mathrm{mg} \mathrm{ml}^{-1}\right)$. After autoassembly ( $20 \mathrm{~min}$ in darkness) the sample was divided and each portion irradiated with $731 \mathrm{~nm}$ (far-red, left) or $657 \mathrm{~nm}$ (red, right) light. Note the blue or green transition associated with phytochrome photoconversion. c, Absorbance characteristics of phy* after irradiation with saturating red (R) or far-red (FR) light, and the calculated difference spectrum. tively, and an isosbestic point at $677 \mathrm{~nm}$.

This is the first report of a spectrally functional prokaryotic phytochrome. In most lower organisms light is detected by retinal or coumaric acid-based photoreceptors. However, a Fremyella gene, rcaE, involved in chromatic adaptation and encoding a putative histidine-kinase sensor protein was recently described ${ }^{13}$. Although the conceptual gene product shows local similarities to PHYE in Arabidopsis, no significant homology to the phytochrome chromophore-binding domain is apparent ${ }^{13}$ and photoreceptor activity has yet to be demonstrated. The Synechocystis phy gene product is the most phytochrome-like of the Synechocystis genome and among all known prokaryotic sequences.

A simple prokaryote has advantages for basic studies of phytochrome biology. For example, co-expression in the heterologous E. coli host might be useful in studying subsequent components of the signal transduction pathway. Furthermore, milligram amounts of homogeneous, spectrally active Synechocystis phytochrome holoprotein can be produced easily in our system. As concentrations of $10 \mathrm{mg} \mathrm{ml}^{-1}$ can be achieved readily - at least ten times higher than in other phytochrome overexpression systems known to us - there remains no barrier in principle to obtaining crystals for X-ray diffraction analysis of phytochrome molecular structure. Jon Hughes, Tilman Lamparter

\section{Franz Mittmann, Elmar Hartmann}

Institut für Pflanzenphysiologie und Mikrobiologie, Freie Universität Berlin,

Königin-Luise-Strasse 12-16,

D-14195 Berlin, Germany

e-mail: hughes@zedat.fu-berlin.de

\section{Wolfgang Gärtner}

Max-Planck-Institut für Strahlenchemie,

Postfach 101365, D-45413 Mülheim/Ruhr, Germany Annegret Wilde, Thomas Börner

Institut für Biologie, Humboldt Universität Berlin,

Chausseestrasse 117, D-10115 Berlin, Germany

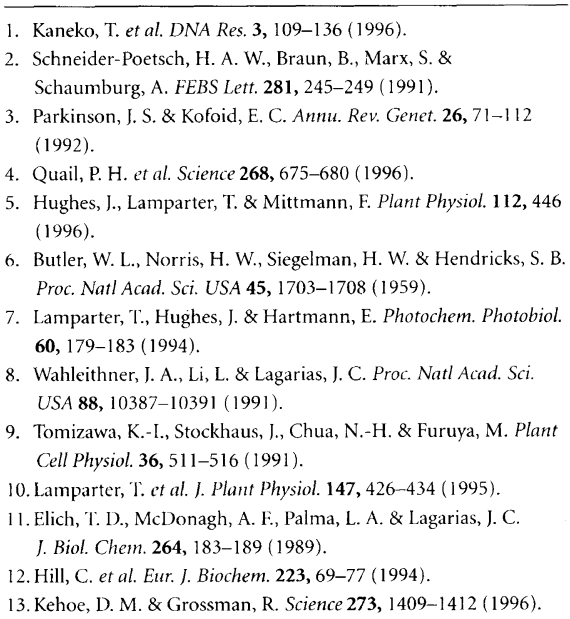

